\title{
Preoperative interventional artery embolization for the treatment of a giant malignant phyllodes tumor: A case report
}

\author{
YUE TIAN $^{1,2^{*}}$, LINLIN LIU ${ }^{3 *}$, LIANG CHEN ${ }^{2}$, SHENGDI ZHAO ${ }^{1,2}$, RUIJUN SU $^{1,2}$, \\ WENZHU ZHANG ${ }^{1,2}$, AIMEI JIANG ${ }^{1,2}$, WENLIN CHEN ${ }^{4}$ and FEI GE ${ }^{2}$ \\ ${ }^{1}$ No. 1 School of Clinical Medicine, Kunming Medical University; ${ }^{2}$ First Affiliated Hospital of \\ Kunming Medical University, Kunming, Yunnan 650032; ${ }^{3}$ School of Forensic Medicine, Kunming Medical University; \\ ${ }^{4}$ The Third Affiliated Hospital of Kunming Medical University, Kunming, Yunnan 650118, P.R. China
}

Received August 27, 2020; Accepted April 1,2021

DOI: $10.3892 /$ mco.2021.2295

\begin{abstract}
Phyllodes tumors (PTs) are rare but complex fibroepithelial lesions of the breast. The present report describes an unusual case of a giant malignant PT with a rich blood supply treated with dominant blood supply internal thoracic artery interventional embolization before surgery. A 41-year-old woman without underlying systemic disease presented with a tumor $>20 \mathrm{~cm}$ in diameter growing rapidly in the left breast. Radiological results indicated a giant circular tumor with a clear boundary occupying the whole breast, possible invasion of the major pectoralis muscle and several enlarged lymph nodes in the left axillary region. Computed tomography angiography showed a large mass with a rich and powerful blood vessel supply and preoperative interventional embolization was performed to block the internal thoracic artery. Three days after artery embolization, mastectomy and grade I axillary lymph node dissection were performed. The giant tumor measured $17 \times 16 \times 11 \mathrm{~cm}$. The surgery successfully treated the pain and tumor necrosis and the patient received chemotherapy and local radiotherapy. No recurrence was found at the 14-month follow-up.
\end{abstract}

Correspondence to: Professor Wenlin Chen, The Third Affiliated Hospital of Kunming Medical University, 519 Kunzhou Road, Xishan, Kunming, Yunnan 650118, P.R. China

E-mail: chenwenlin@hotmail.com

Professor Fei Ge, First Affiliated Hospital of Kunming Medical University, 295 Xichang Road, Kunming, Yunnan 650032, P.R. China

E-mail: ajqnadjd@hotmail.com

${ }^{*}$ Contributed equally

Abbreviations: $\mathrm{PT}$, phyllodes tumor; BCS, breast-conserving surgery; CNB, core needle biopsy; CTC, circulating tumor cell

Key words: breast tumor, malignant phyllodes tumor, interventional artery embolization, preoperative

\section{Introduction}

The American Cancer Society estimates that $10 \%$ of women will have a detectable breast mass in their lifetime (1). A phyllodes tumor (PT) of the breast is a rare fibroepithelial neoplasm, accounting for $0.3-1.0 \%$ of all breast tumors (2). PTs may occur at any age but are commonly observed in women aged between 40 and 50 years (3). PTs can recur locally and rarely metastasize, and only a few become malignant $(\sim 10 \%)(4,5)$. Whether benign or malignant, certain PTs grow rapidly, even to a diameter $>10 \mathrm{~cm}$ (6). Because the clinical outcomes of all PTs range from local recurrence in 6.3-32.0\% of patients to metastasis in $1.7-40.0 \%$ of patients, the National Comprehensive Cancer Network guidelines recommend complete surgical resection of PTs, particularly malignant PTs, to obtain a negative margin $\geq 1 \mathrm{~cm}$ (7). However, the scope of surgery [breast-conserving surgery (BCS) vs. mastectomy] and role of adjuvant radiation therapy have been controversial (8). The initial surgical removal of a giant PT is often challenging (6). Numerous studies have shown that preoperative tumor reduction improves the success rate of breast cancer surgery and results in good prognosis $(6,9)$. The present study reports an unusual case of a giant malignant PT of the breast (MPTB) with a rich blood supply. Preoperative interventional embolization without arterial infusion chemotherapy and and protective embolization on extrahepatic branches, such as the internal mammary artery, were performed. Following arterial embolization, the heart rate gradually decreased to a normal level, tumor surface necrosis quickly appeared and the patient exhibited no fever, chest pain or other symptoms; surgery successfully treated the pain and tumor necrosis. The patient was transferred to chemoradiotherapy from local radiotherapy. No recurrence was found at the 14-month follow-up. Written informed consent was obtained from the patient.

\section{Case report}

A previously healthy 41-year-old woman without underlying systemic disease detected an olive-sized lump in her left breast 6 months before hospital admission. The lump grew rapidly, with itching and peeling breast skin observed after $\sim 1$ month; less than half a month prior to admission, a skin ulceration appeared on the left breast. The patient had no family history 
A

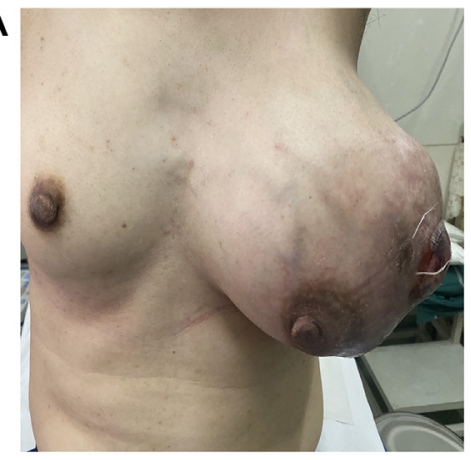

C

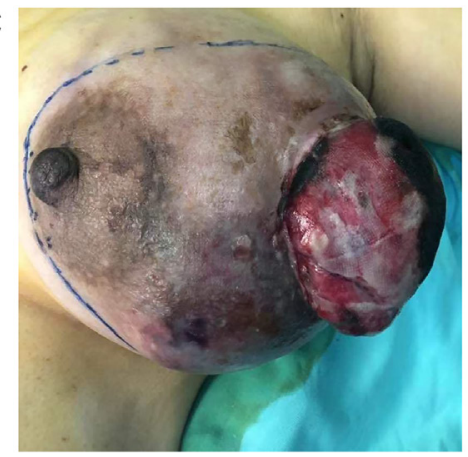

B

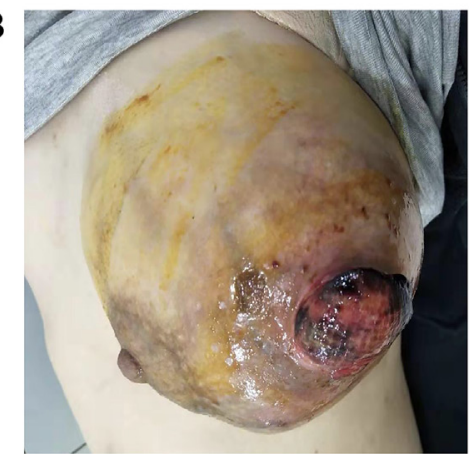

D

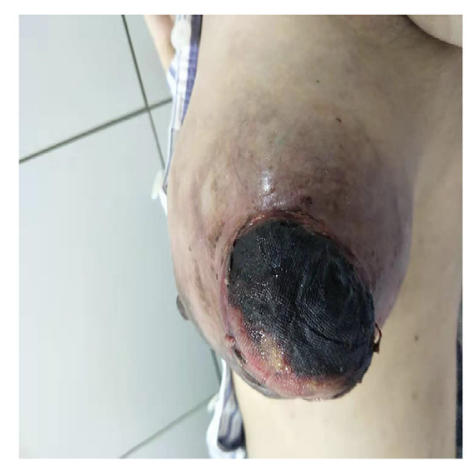

Figure 1. Anteroposterior and lateral radiographs of the tumor before and after embolization. (A) Anteroposterior and (B) lateral film were acquired before and after core needle biopsy. (C) Anteroposterior and (D) lateral film were taken 3 days after embolization. Following embolization, the growth rate of the tumor decreased and the skin of the nipple and areola and tumor surface became ischemic and necrotic.

A

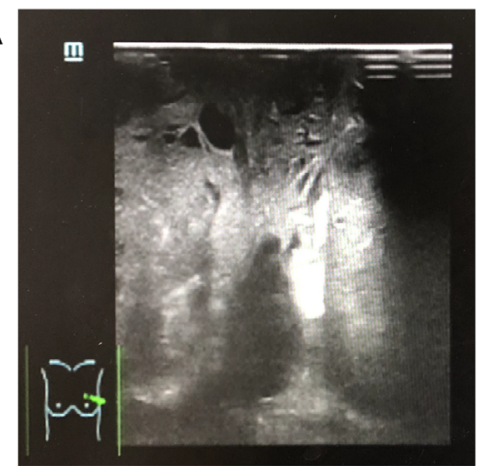

C

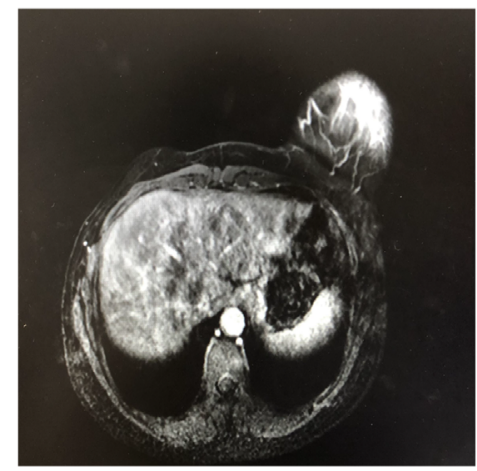

B

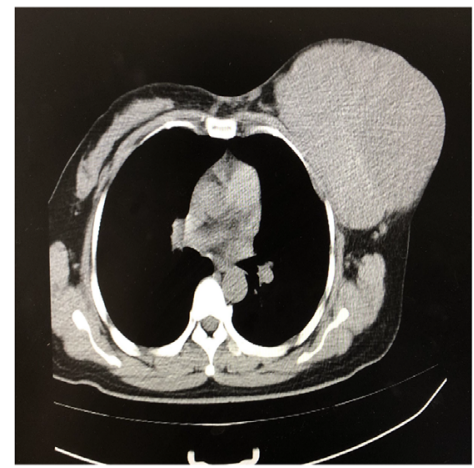

D

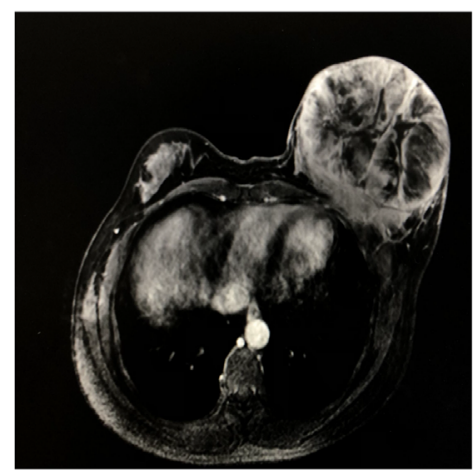

Figure 2. Imaging examination results of the tumor. (A) Color Doppler ultrasound and (B) T showed a giant circular tumor with a clear boundary occupying the left breast. (C and D) MRI showed possible invasion of the major pectoralis muscle.

of breast cancer or childbearing. Upon admission to The First Affiliated Hospital of Kunming Medical University, the tumor measured $>20 \mathrm{~cm}$ in diameter and was larger than the right breast and the ulceration measured $2 \mathrm{~cm}$ in diameter; the engorgement of multiple veins was observed in the subcuta- neous tissue (Fig. 1A and B). Palpation revealed that the tumor was slightly hard and elastic, and certain areas were fluctuant. Because of hypermetabolism and chronic blood loss from the tumor, the patient's heart rate increased to 128 beats per minute. 

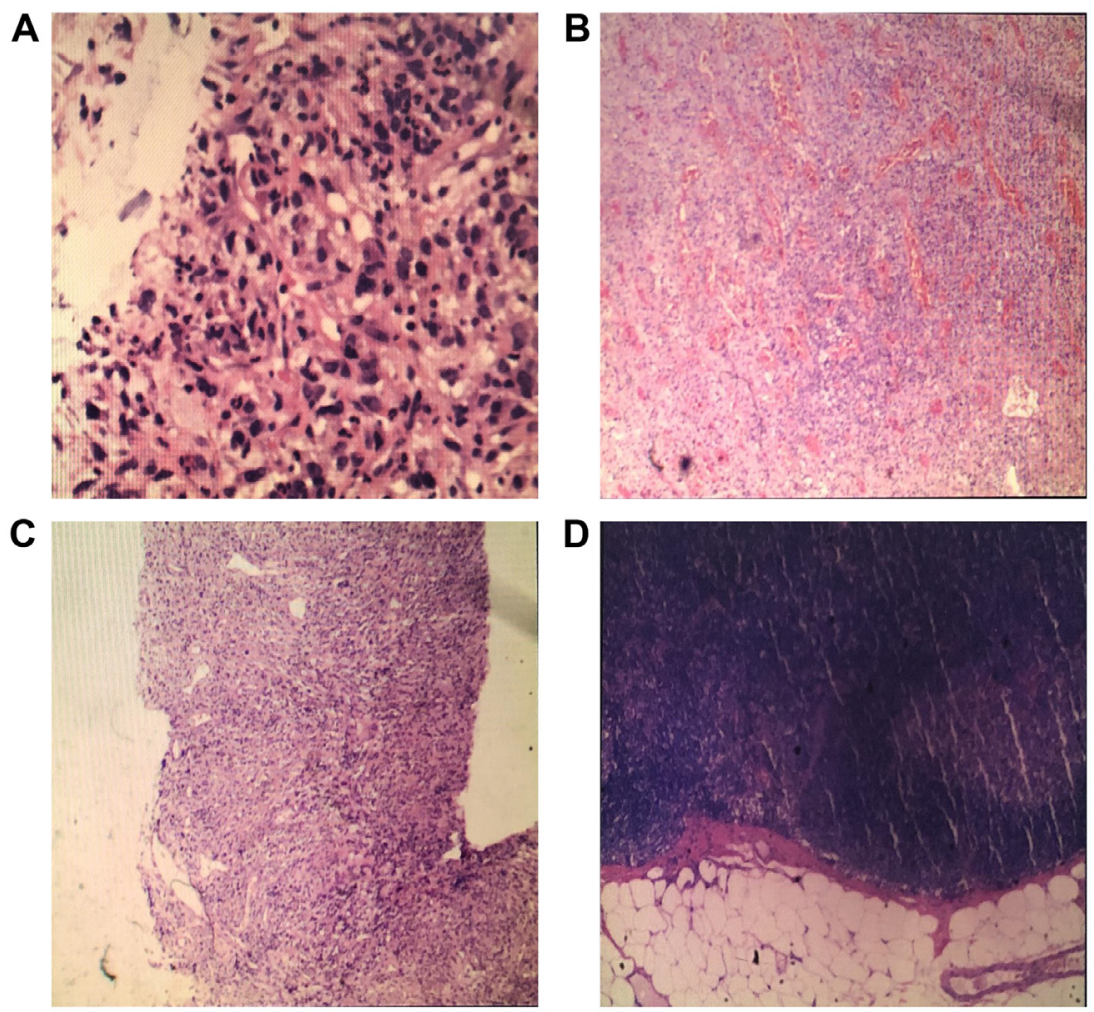

Figure 3. Pathological examination of the tumor. (A) Preoperative core needle biopsy identified the tumor as malignant myxofibrosarcoma.(B and C) Postoperative histology of the tumor. The malignant phyllodes tumor measured 17x16x11 cm. (D) Left axillary lymph node; 11 lymph nodes were found, with no tumor metastasis.

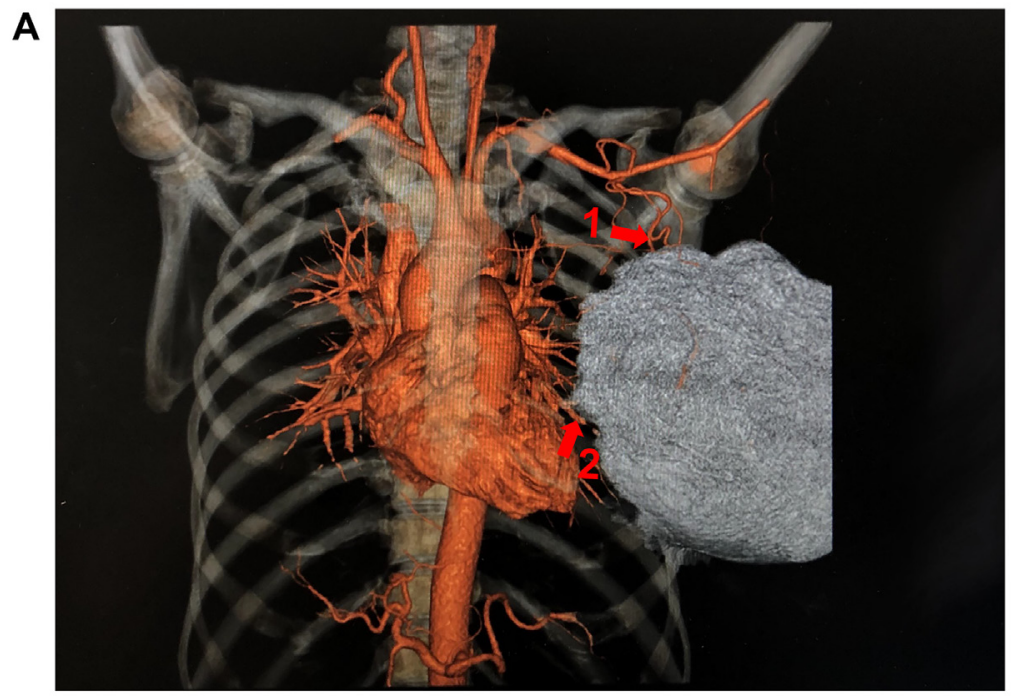

B

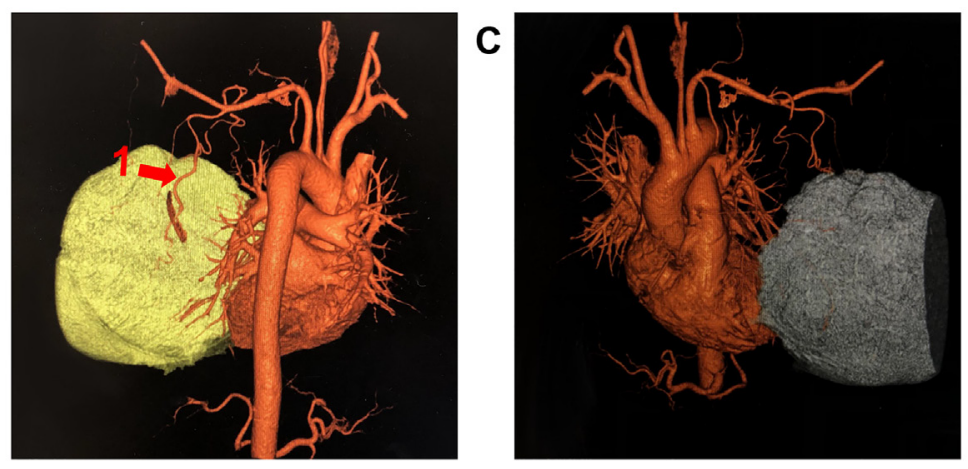

Figure 4.CT angiography shows a large mass with a rich and powerful blood vessel supply.(A) Whole,(B) back and (C) front view of the tumor blood supply. Arrows 1 and 2 indicate the lateral and internal thoracic arteries, respectively. 

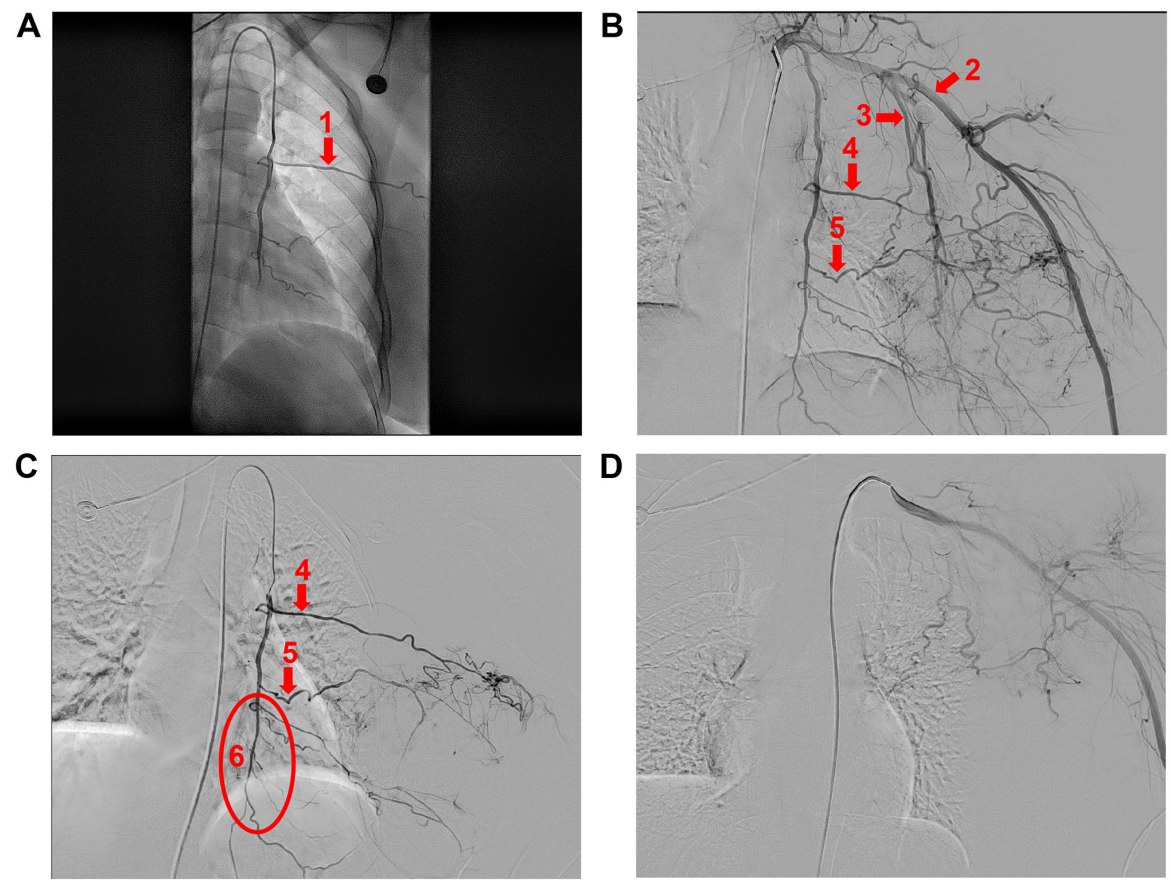

Figure 5. Arteriography identifying the vascular blood supply to the tumor and performance of embolization. A single curved catheter was used to hook the left subclavian artery for angiography, with superselective access to the internal and external thoracic arteries, and a gelatin sponge was used to protect the distal end of the internal thoracic artery. (A) Superselective access to the internal thoracic artery (arrow 1). (B) Angiography showed that the primary blood supply arteries of the tumor were the branches of the left internal thoracic artery and left subclavian artery (arrow 2). Arrow 3 indicates the external thoracic artery; arrows 4 and 5 indicate the intercostal artery. (C) Branches of the internal thoracic artery were embolized using gelatin sponge particles and protective embolization of the distal internal thoracic artery (arrow 6) was performed using a gelatin sponge. (D) Completed embolization.
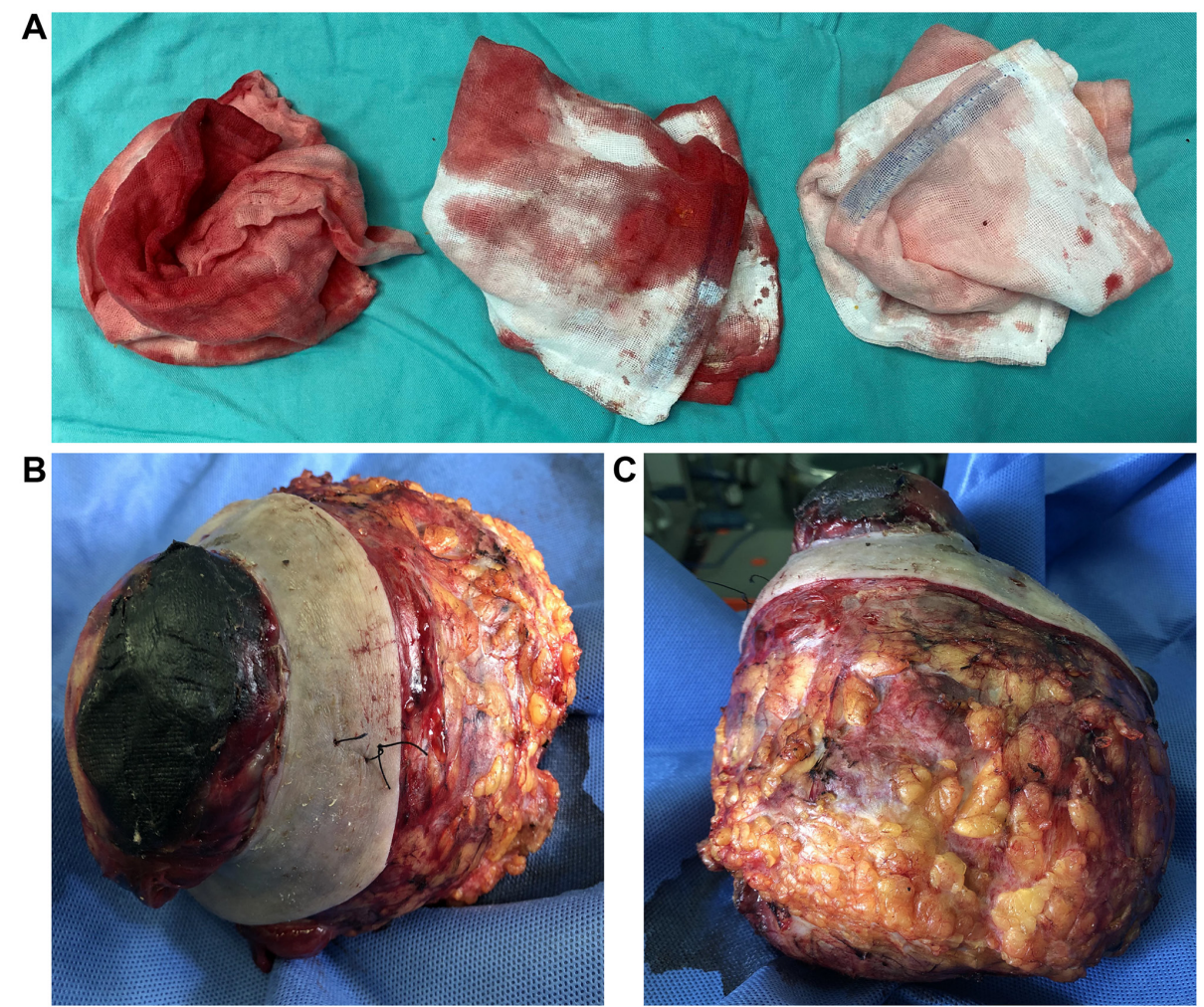

Figure 6. Intraoperative bleeding and appearance of the tumor. (A) Amount of bleeding was $\sim 50 \mathrm{ml}$, and $<80 \mathrm{ml}$ during the surgery. (B) and (C) show the appearance of the removed tumor. Measurement of huge tumor was $17 \times 16 \times 11 \mathrm{~cm}$.

Color Doppler ultrasound revealed a very large heterogeneous echo in the left mammary gland but could not accurately measure the size of the lesion, which was $\sim 192.6 \times 117.2 \mathrm{~mm}$. The boundary was obscure and the form was irregular, with a 

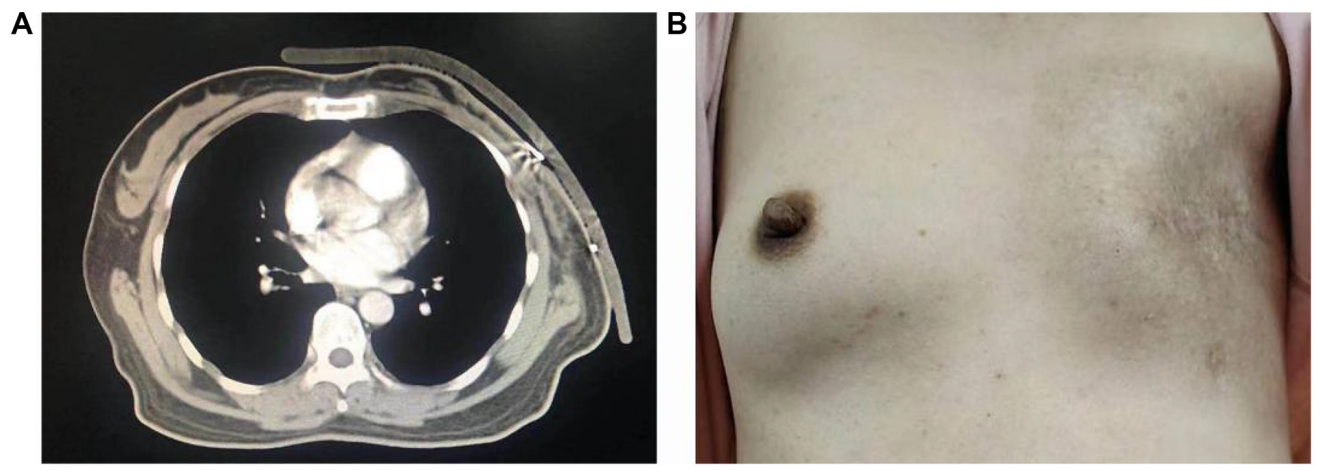

Figure 7. CT scan before postoperative radiotherapy and photos at the 14-month follow-up. (A) Srgical scar was signed by a galvanized wire on CT scan before local radiotherapy. (B) Follow-up image of the patient after 14 months.

rich blood supply inside. Multiple lymph nodes were enlarged and rounded and hypoechoic nodules were observed in the left axillary region (Fig. 2A). Computed tomography (CT) showed a giant tumor occupying the left breast and suspected invasion of the major pectoralis muscle, which indicated that the tumor was potentially malignant (Fig. 2B). MRI showed a giant circular tumor with a clear boundary occupying the whole breast and a partially patchy unenhanced zone in the center measuring $\sim 13 \times 13 \times 14 \mathrm{~cm}$ (upper and lower diameter x left and right diameter $x$ before and after diameter). MRI also revealed possible invasion of the major pectoralis muscle and several enlarged lymph nodes in the left axillary region, the largest of which measured $\sim 1.6 \times 0.8 \mathrm{~cm}$. No abnormality was observed in the entire right breast tissue (Fig. 2C and D). Preoperative core needle biopsy $(\mathrm{CNB})$ revealed that the biopsy specimen of the left breast was malignant myxofibrosarcoma. The immunohistochemical report was as follows: SMA (+), Des (-), KI-67 (+)30\%, CK5/6 (-/+), P63 (-), CK (-) and VIM (+) (Fig. 3A). Additionally, the patient received comprehensive CT and MRI examination; the results showed that the brain, lung, liver, bones and other organs exhibited no signs of metastasis.

The tumor measured $24 \mathrm{~cm}$ in diameter; the fungating wound was $5 \mathrm{~cm}$ above the breast surface and measured $5 \mathrm{~cm}$ in diameter on the day before surgery. Because of the rich blood vessel supply revealed by MRI, the patient underwent CT angiography of the breast tumor, which revealed that the perforating branch of the left internal thoracic artery and branch of the left subclavian artery were the dominant feeding arteries transporting blood to the tumor in the left breast. These vessels were disordered and tangled (Fig. 4). During the hospital examination, the tumor grew rapidly with bleeding. In order to slow tumor growth, decrease intraoperative blood loss and improve the success rate of the operation, preoperative dominant blood supply internal thoracic artery interventional embolization was performed, and surgery was delayed.

The patient underwent percutaneous arterial embolization, performed by an interventional physician, resulting in tumor necrosis and contraction by limiting the tumor blood supply. The process was performed as follows: After administering local anesthesia, a 5F arterial sheath was inserted into the right femoral artery using the Seldinger puncture technique. An elbowed catheter was used to image the left subclavian artery; abnormal staining was observed in the tumor, superselective to the internal and external thoracic arteries (Fig. 5A and B). Protective embolization was performed distal to the internal thoracic artery using a gelatin sponge (Fig. 5C), and the 5F arterial sheath was replaced with a $2.6 \mathrm{~F}$ micropipe superselective to the dominant blood-supplying arteries guided by a 0.018 -inch micro godet. Next, gelatin sponge granules (710-1,000 $\mu \mathrm{m})$ were injected; imaging revealed that most of the staining in the tumor had disappeared (Fig. 5D). Following artery embolization, fever and black necrotic tissue at the tumor site were observed. Fever resolved within $24 \mathrm{~h}$, but the area of necrosis on the tumor site expanded. No other serious complications were observed and the tumor stopped growing following artery embolization (Fig. 1C and D).

Three days after artery embolization, the patient underwent surgery. Although axillary metastases of PTs are rare, mastectomy and level I axillary lymph node excision due to swelling were performed for safety after discussion. The tumor measured 17x16x11 cm. The bleeding volume during the operation was $<80 \mathrm{ml}$ and the elasticity of several vessels was lost. The whole process lasted $1.5 \mathrm{~h}$ (Fig. 6).

The postoperative biopsy specimen was diagnosed as a malignant tumor, specifically myxofibrosarcoma (Fig. 3B and C). The tumor measured 17x16x11 cm, and the following parameters were noted: Nerve invasion (-), vascular invasion (-), invisible edge of the breast (-), invisible edge of the base (-), invisible edge of the skin (-), nipple (-) and skin (-). The immunohistochemical report was as follows: SMA (+), Des (-), KI-67 (+)30\%, CK5/6 (+), P63 (-), CK (-), and VIM (+). A total of 11 lymph nodes were found in the left axillary gland, with no metastatic tumors (Fig. 3D).

The surgery successfully treated the pain and tumor necrosis (Fig. 7A). Postoperatively, the patient recovered well and the incision healed desirably. After surgery, it was recommended that the patient undergo circulating tumor cell (CTC) detection and whole-genome sequencing (WGS); CTC analysis was negative. Since the tumor invaded the muscle layer of the chest wall, it was hypothesized that the relevant microscopic lesion may still be present. Therefore, we recommend that patients receive concurrent chemoradiotherapy to prevent recurrence following surgery. The patient was treated with local radiotherapy 18 times $(3 \mathrm{~Gy} /$ time, total dose $54 \mathrm{~Gy})$ and with apatinib (250 mg/day) on the first and fourth day and oral ticgio (40 mg/day). The patient refused further chemotherapy and did not accept a recommendation 
for antiangiogenic drugs. No recurrence was found at the 14-month follow-up (Fig. 7B).

\section{Discussion}

PTs are rare but complex fibroepithelial lesions of the breast that are classified as benign, borderline or malignant (5). PTs and malignant PTs present significant challenges in diagnosis, classification, predictive behavior and clinical treatment (10). From a diagnostic and management perspective, correctly identifying malignant PTs is critical. They should be surgically eradicated and effectively treated at the time of diagnosis because the risks of metastasis and death from such tumors, although relatively small, are well-established (5).

PTs have non-specific clinical and radiological manifestations that can easily be confused with those of other similar breast masses (11). Clinical and radiological diagnoses are difficult, requiring joint confirmation from multiple assessments. In the present case, color Doppler ultrasound showed feature information that could assist in diagnosis. CT showed whether lung metastasis occurred and infiltration was assessed by MRI. However, these methods cannot help to make a definitive preoperative diagnosis. CNB is a widely used, highly sensitive method to obtain a preoperative diagnosis of breast cancer (12), although the tissue obtained in CNB does not represent the whole tumor. Here, these results and clinical appearance were combined, and the diagnosis was confirmed.

Surgical excision is the preferred procedure because it can obtain a negative margin in the case of a final diagnosis of a malignant PT $(5,11)$. Although mastectomy is the only treatment method for large tumors, the extent of surgery (BCS vs. mastectomy) for small tumors remains controversial (8). Though axillary lymph node metastasis is rare, some cases of giant PT-mixed breast cancer have been reported. In the present case, the tumor grew rapidly and was very large. The patient had metabolic consumption and risks were associated with surgery.

Interventional embolization was developed to manage vascular areas of bleeding, including in the brain, lung and liver (13) and is an effective and safe choice (14). Vascular occlusion by embolization has also been used to control severe or recurrent bleeding in fungal breast cancer (15). In the case of sternal erosion of breast cancer, embolization can effectively control bleeding and save lives (16). Interventional embolization is used to decrease the preoperative breast tumor volume and resection is performed 23 days after embolization. Embolization effectively decreases intraoperative bleeding and improves surgical results (16). In the present case, the left breast tumor was primarily supplied by the left internal thoracic artery branch and left internal thoracic artery perforator. The blood supply vessels were thickened, the blood supply was rich and the tumor grew rapidly with bleeding. Therefore, catheter embolization was performed before surgery to slow tumor growth and decrease intraoperative bleeding. Three days after embolization, resection was successfully performed. No clear guidance exists to determine the operation time after embolization, and the operation should be performed carefully according to the tumor growth rate, bleeding and ulceration for each patient. Complications of interventional embolization are rare (14), and no complications were observed in the present case. However, potential complications associated with this type of operation, such as infection after tumor necrosis, distal embolization and cerebral infarction (9), should be carefully monitored during the process.

The precise role of adjuvant radiotherapy remains debatable, but it has been personalized for certain patients with malignant PT. Although radiotherapy has no significant effect on survival rate, it can decrease local recurrence (5). When patients do not receive radiation and chemotherapy, they may obtain passive results. For example, Kuo et al (6) described patients who received preoperative embolization chemotherapy and successful tumor suppression. However, chemotherapy is not an option for patients two months after recurrence (6). In the present case, since the tumor invaded the muscle layer of the chest wall, it was recommended that the patient receive chemotherapy and local radiotherapy to prevent recurrence; the 14-month follow-up after chemoradiotherapy was good. This result suggests that, in the case of an MPTB, particularly an MPTB with chest wall invasion, chemoradiotherapy is necessary.

WGS and CTC detection lead to high-resolution analysis of cancers and reliably guided personalized therapy (17); the patient accepted CTC detection but rejected WGS. The CTC detection results were negative.

Patients with large lesions are inclined to undergo mastectomy. In cases of giant malignant PTs, preoperative intervention artery embolization can inhibit tumor growth, decrease bleeding during an operation, increase the operation speed and shorten the period of postoperative convalescence. Preoperative intervention artery embolization was effective and safe for the present patient, and postoperative local radiotherapy may be necessary for a good prognosis.

\section{Acknowledgements}

Not applicable.

\section{Funding}

The present report was supported by the National Natural Science Foundation of China (grant no. 81660438).

\section{Availability of data and materials}

The datasets used and/or analyzed during the current study are available from the corresponding author on reasonable request.

\section{Authors' contributions}

FG, AJ and WC conceived and designed the study. YT, LC, SZ, RS, WZ and LL performed data acquisition and analysis. YT, LL and FG drafted the manuscript. FG and LL reviewed the manuscript. All the authors read and approved the final manuscript.

\section{Ethics approval and consent to participate}

Approval was obtained from the ethics committee of Kunming Medical University. The procedures performed in this study 
adhered to the tenets of the Declaration of Helsinki. Written informed consent was obtained from the patient.

\section{Patient consent for publication}

Written informed consent was obtained from the patient included in the study.

\section{Competing interests}

The authors declare that they have no competing interests.

\section{References}

1. DeSantis C, Ma J, Bryan L and Jemal A: Breast cancer statistics, 2013. CA Cancer J Clin 64: 52-62, 2014.

2. Chang J, Denham L, Dong EK, Malek K and Lum SS: Trends in the diagnosis of phyllodes tumors and fibroadenomas before and after release of WHO classification standards. Ann Surg Oncol 25: 3088-3095, 2018

3. Krings G, Bean GR and Chen YY: Fibroepithelial lesions: The WHO spectrum. Semin Diagn Pathol 34: 438-452, 2017.

4. Tan PH, Ellis I, Allison K, Brogi E, Fox SB, Lakhani S, Lazar AJ, Morris EA, Sahin A, Salgado R, et al: The 2019 WHO classification of tumours of the breast. Histopathology 77 : $181-185,2020$

5. Tan BY, Acs G, Apple SK, Badve S, Bleiweiss IJ, Brogi E, Calvo JP, Dabbs DJ, Ellis IO, Eusebi V, et al: Phyllodes tumours of the breast: A consensus review. Histopathology 68 : $5-21,2016$

6. Kuo CY, Lin SH, Lee KD, Cheng SJ, Chu JS and Tu SH: Transcatheter arterial chemoembolization improves the resectability of malignant breast phyllodes tumor with angiosarcoma component: A case report. BMC Surg 19: 100, 2019.

7. Gradishar WJ, Anderson BO, Balassanian R, Blair SL, Burstein HJ, Cyr A, Elias AD, Farrar WB, Forero A Giordano SH, et al: Breast cancer, version 4.2017, NCCN clinical practice guidelines in oncology. J Natl Compr Canc Netw 16: 310-320, 2018
8. Mitus J, Reinfuss M, Mitus JW, Jakubowicz J, Blecharz P, Wysocki WW and Skotnicki P: Malignant phyllodes tumor of the breast: Treatment and prognosis. Breast J 20: 639-644, 2014.

9. Hashimoto K, Mimura H, Arai Y, Doi M, Kojima Y, Tsugawa $\mathrm{K}$ and Nakajima Y: Successful preoperative chemoembolization in the treatment of a giant malignant phyllodes tumor. Cardiovasc Intervent Radiol 39: 1070-1075, 2016.

10. Moten AS and Goldberg AJ: Malignant phyllodes tumors of the breast: Association between race, clinical features, and outcomes J Surg Res 239: 278-283, 2019.

11. Atalay C, Kınaş V and Çelebioğlu S: Analysis of patients with phyllodes tumor of the breast. Ulus Cerrahi Derg 30: 129-132, 2014.

12. Dillon MF, Quinn CM, McDermott EW, O'Doherty A, O'Higgins N and Hill ADK: Needle core biopsy in the diagnosis of phyllodes neoplasm. Surgery 140: 779-784, 2006.

13. Moriarty JM, Xing MZ and Loh CT: Particle embolization to control life-threatening hemorrhage from a fungating locally advanced breast carcinoma: A case report. J Med Case Rep 6: 186,2012

14. Corvino F, Giurazza F, Cangiano G, Cavaglià E, Amodio F, Magistris GD, Corvino A and Niola R: Safety and effectiveness of transcatheter embolization in the treatment of internal mammary artery injuries. Radiol Med 123: 369-377, 2018.

15. Aksoy S, Akçe B, Kiliçkesmez Ö, Gürsü RU, Cakır MS, Nazlı MA and Aren A: Transcatheter arterial embolization for controlling severe bleeding from recurrent locally-advanced breast cancer. J Breast Health 12: 137-140, 2016.

16. Leung JWT, Gotway MB and Sickles EA: Preoperative embolization of vascular phyllodes tumor of the breast. AJR Am J Roentgenol 184 (Suppl 3): S115-S117, 2005.

17. Gulbahce N, Magbanua MJM, Chin R, Agarwal MR, Luo XH, Liu J, Hayden DM, Mao Q, Ciotlos S, Li ZY, et al: Quantitative whole genome sequencing of circulating tumor cells enables personalized combination therapy of metastatic cancer. Cancer Res 77: 4530-4541, 2017. International (CC BY-NC-ND 4.0) License. 